\title{
Trayectorias docentes
}

Departamento de Formación y Actualización Académica

\section{Introducción}

\begin{abstract}
A lo largo de 43 años de existencia, la Universidad Autónoma de Aguascalientes (UAA) ha dado paso al desarrollo de cientos de profesores de diversas áreas del conocimiento, quienes por medio de su labor en la docencia, la investigación y la difusión de la cultura y del conocimiento han retribuido a la confianza que la sociedad les ha conferido como actores educativos centrales de la institución, coadyuvando en la formación de los profesionistas que ahora impulsan el avance social y económico de la localidad, del estado y del país.

Con el objetivo de acercarnos a conocer algunas de las muchas historias, anécdotas, retos y logros alcanzados en su ejercicio profesional por más de 30 años de servicio como docentes, en estas líneas se esbozan las trayectorias de tres profesores de la UAA, las cuales fueron compartidas en entrevistas sostenidas en el programa radiofónico semanal "El Gis", del Departamento de Formación y Actualización Académica (DEFAA), de la Dirección General de Docencia de Pregrado.
\end{abstract}

Para seguir el curso de estas tres trayectorias, en las dimensiones generales comprendidas para la labor docente en el contexto institucional (la personal, la pedagógica y la disciplinar) se considera importante referirse al origen de la palabra trayecto o trayectoria (trajectus), que deriva del latín: trans que significa al otro lado de, a través de, más allá, y, de jácere que significa arrojar (Mateos, 2005: 287); asimismo, definida por la Real Academia Española (2015), como la "línea descrita en el plano o en el espacio por un cuerpo en movimiento" o, como el "curso que, a lo largo del tiempo, sigue el comportamiento o el ser de una persona, de un grupo social o de una institución".

Es así que el desarrollo de la profesión como docente, a lo largo del tiempo, logra trascender más allá de sólo recorrer el camino de un punto a otro, si se desea sumar proyectos concretados, metas alcanzadas y experiencias de vida para el crecimiento personal. La labor docente requiere de la constancia, la perseverancia, la convicción y la diversificación de funciones, que demandan al profesor una formación integral tanto en el desarrollo personal, como en su formación pedagógica y disciplinar, a fin de afrontar los compromisos que la profesión le demande. Lograr el desarrollo equilibrado en estas dimensiones ha sido posible para estos profesores por su amor, empeño y pasión puestos en cada actividad, proyecto y enseñanza transmitida a sus estudiantes.

Los testimonios se presentan a continuación en orden cronológico, de acuerdo con la realización de las entrevistas, en el programa radiofónico "El Gis"; "un espacio de interacción y reflexión

1 Actualmente, se transmite por Radio Universidad 94.5 de FM, todos los miércoles de 10:30 a 11:00 hrs. Puede conocerse más sobre el programa en: http://www.uaa. $\mathrm{mx/direcciones/dgdp/defaa/elgis.html.}$ 


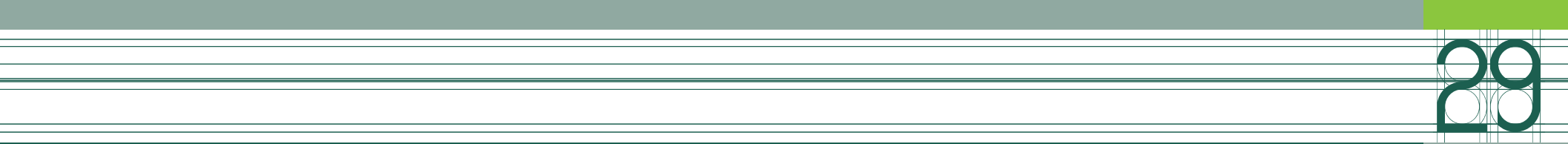

sobre temáticas relacionadas al quehacer educativo" (UAA, 2015: 3) y propicio para el acercamiento entre la comunidad docente y el público en general de la entidad, principalmente; cuenta con más de 1000 programas emitidos, y una trayectoria de 22 años al aire.

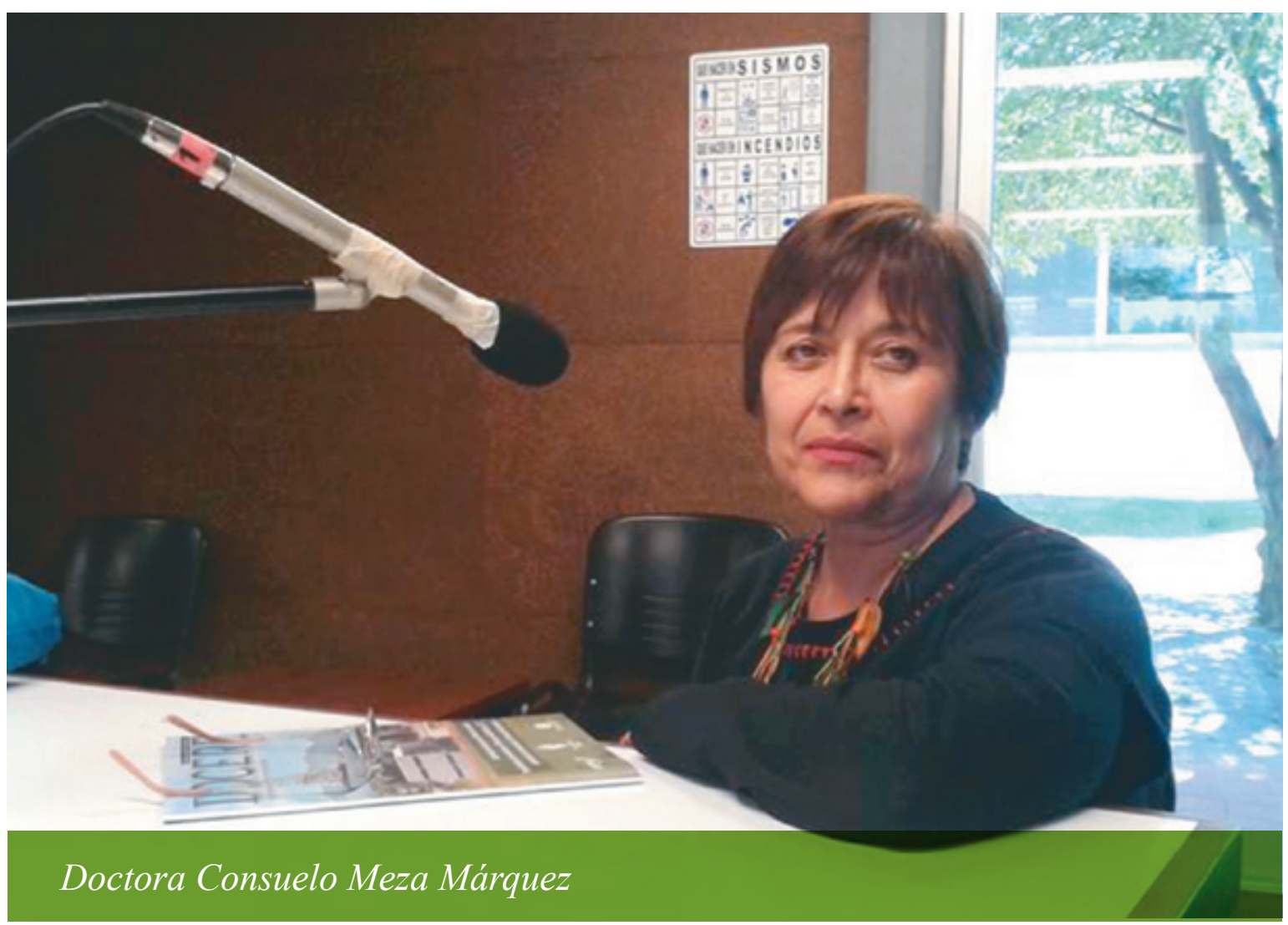

$\mathrm{E}$ s profesora investigadora de dedicación exclusiva, adscrita al Departamento de Sociología y Antropología, del Centro de Ciencias Sociales y Humanidades, desde hace 31 años. Durante ese trayecto, también se ha desempeñado como Jefa del Departamento de Sociología y Antropología, y como Secretaria de Docencia de Pregrado en la UAA, entre otros cargos. Además ha sido miembro del Sistema Nacional de Investigadores (SNI).

Originaria de Ciudad Juárez, Chihuahua, emigró de su tierra natal para cursar la licenciatura en Sociología en la Universidad Autónoma de Aguascalientes, inspirada por el contraste que observaba entre la cultura de su ciudad y El Paso Texas, donde hizo sus estudios de primaria, secundaria y bachillerato (High school). Posteriormente, estudió la Maestría en Investigación en Ciencias Sociales y Humanidades en la UAA, y realizó dos especialidades en el Colegio de México, una en Estudios de la Cultura y la otra en Estudios de la Mujer. Sus estudios de doctorado los realizó en la Universidad Autónoma Metropolitana de Iztapalapa (UAM), obteniendo el grado de Doctora en Humanidades en Teoría Literaria. 
Durante ese trayecto ha radicado en la Ciudad de México y en otros países como Guatemala, donde desarrolló gran parte de sus investigaciones, las cuales ha orientado principalmente a las líneas de crítica social y literaria feminista, y de identidad y cultura de equidad.

Aunque se inició en la investigación por el estudio de la transferencia de la tecnología en comunidades rurales, en Pabellón de Hidalgo, Aguascalientes, comentó: "con la llegada de las empresas transnacionales, la agricultura como actividad económica desapareció. Eso fue más o menos paralelo a asumir el cargo como Jefa del Departamento de Sociología y Antropología, [por lo cual] durante esos seis años no podía hacer el trabajo de campo que realicé antes". Derivado de ello y a su pasión por la lectura manifestada desde la niñez, incursionó en el estudio de las mujeres a través de la literatura.

Entre algunos de sus libros publicados ${ }^{2}$ se encuentra: La utopía feminista. Quehacer literario de cuatro narradoras mexicanas contemporáneas, ${ }^{3}$ distribuido por la Asociación Nacional de Universidades e Instituciones de Educación Superior (ANUIES), en la Red Altexto, el cual le ha dado grandes satisfacciones, ha sido multicitado y utilizado como libro de texto en algunas Universidades de Guatemala.

La doctora Consuelo, además de su trabajo académico y de investigación, produce y es conductora del programa radiofónico "De mujeres... símbolo y pensamiento", que se transmite por Radio Universidad (94.5 FM) desde el año de 1991; entre otros objetivos del programa se espera: "que las mujeres hablemos sobre nosotras mismas, que seamos nosotras las que difundamos y transmitamos nuestra propia visión del mundo como producto de la vivencia en un cuerpo sexuado" (Meza, 1998:13).

En cuanto a los aspectos más apasionantes de su labor como docente, considera que:

2 Meza, C. (2012). Diccionario biobibliográfico de narradoras centroamericanas con obra publicada entre 1890 y 2002. México: UAA; (2012). Penélope: antología de cuentistas centroamericanas. Guatemala: F \& G Editores; (2009). Aportaciones para una historia de la literatura de mujeres de América Central. México: UAA; (2007). Narradoras centroamericanas contemporáneas: identidad y crítica socioliteraria feminista. México: UAA.

3 Meza, C. (2000). La utopía feminista. Quehacer literario de cuatro narradoras mexicanas contemporáneas. México: UAA-Uc. Disponible en Google Books: http://bit. Iy/1KQLHC9. el ver cómo lo que una les enseña [a los estudiantes] ha ido fructificando, los sociólogos y los que trabajamos en estudios de género y de la mujer somos sembradores, [parecidos a] los que siembran un árbol, [sabemos] que éste dará fruto después de transcurrido mucho tiempo, y nosotras/nosotros, no vamos a ver ese fruto... no lo vamos a conocer $y$, en ese sentido cada vez que sembramos una semilla, lo tenemos que hacer con la profunda convicción de que se va a dar ese fruto.

Su gran satisfacción durante esta trayectoria ha sido el poder conciliar sus objetivos como madre y como investigadora: "me siento muy contenta, satisfecha, he podido recuperar para mi hija toda esa herencia de sus raíces guatemaltecas, he hecho de ella una mujer fuerte, una mujer segura, con una identidad que le ha dado las herramientas para que pueda defenderse en un futuro".

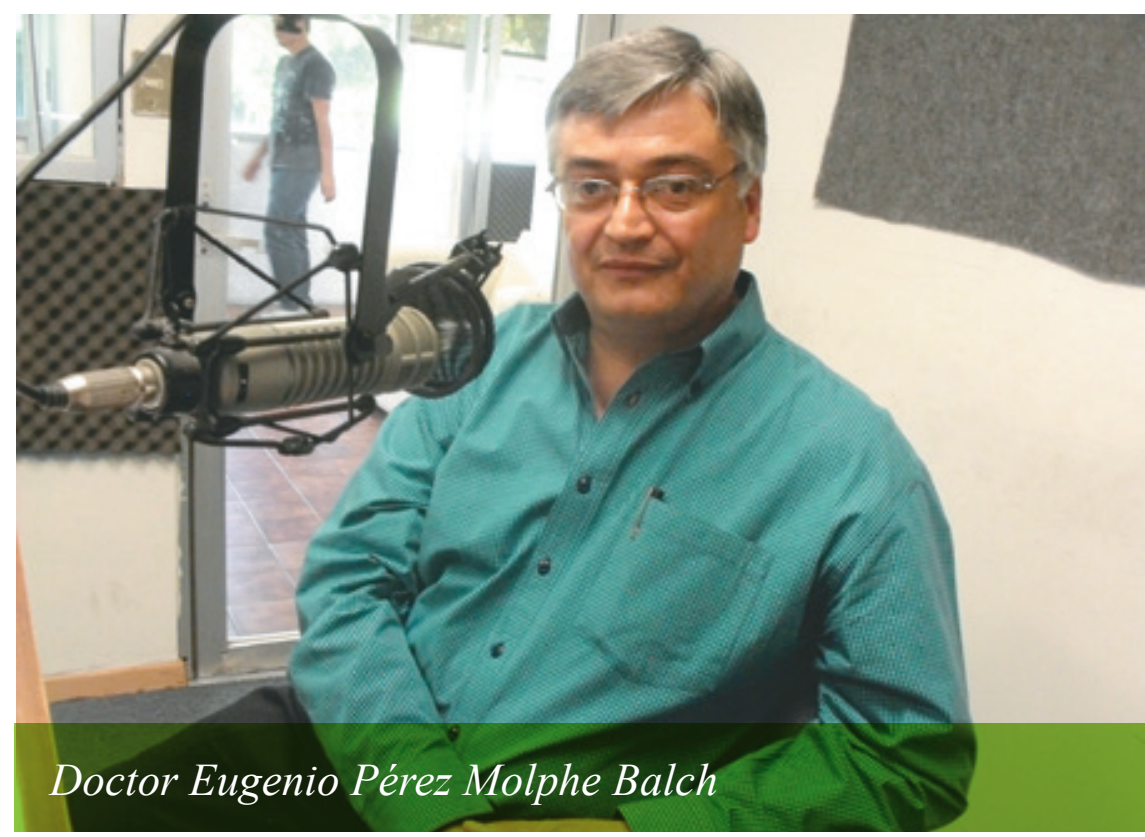

E profesor investigador de tiempo completo, adscrito al Departamento de Química, del Centro de Ciencias Básicas de la Universidad Autónoma de Aguascalientes desde hace 23 años. Es miembro de la H. Junta de Gobierno de la UAA, y pertenece al SNI.

Nació en la ciudad de San Luis Potosí; durante su infancia vivió también en otras ciudades de la república como Culiacán, Sinaloa y México, por el trabajo de su padre. 
Concluidos sus estudios de bachillerato a los 17 años de edad, decidió estudiar la Licenciatura en Biología en la Universidad Autónoma de Aguascalientes, por su gusto e inclinación hacia la investigación científica. Debido a ello continuó su preparación en el Centro de Investigación y de Estudios Avanzados del Instituto Politécnico Nacional, Unidad de Biotecnología e Ingeniería Genética de Plantas, en Irapuato, donde realizó la Maestría en Ciencias en Biología Vegetal, y el Doctorado en Ciencias: Biotecnología de Plantas.

Es padre de dos hijos, de quienes se siente muy orgulloso, los cuales ha criado junto a su esposa originaria de Zacatecas, pero a quien conoció en Aguascalientes durante sus estudios en la licenciatura.

Ha trabajado en líneas de investigación sobre la biotecnología de plantas de zonas áridas, para contribuir a su explotación racional para conservarlas y preservarlas. Asimismo, acerca del desarrollo de plantas transgénicas, árboles frutales resistentes a enfermedades, desarrollo de

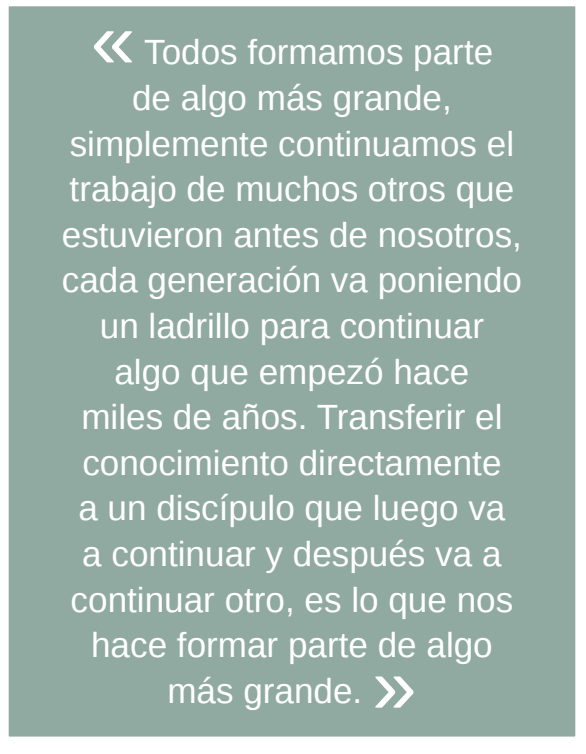

Considera importante reconocer en las relaciones interpersonales que: "todos formamos parte de algo más grande, simplemente continuamos el trabajo de muchos otros que estuvieron antes de nosotros, cada generación va poniendo un ladrillo [...] para continuar algo que empezó hace miles de años [...]. Transferir el conocimiento directamente a un discípulo que luego va a continuar y después va a continuar otro, es lo que nos hace formar parte de algo más grande". Para él, establecer relaciones entre iguales es algo fundamental, por ello considera a sus estudiantes como: "personas con aspiraciones, con virtudes y limitaciones" y, continúa diciendo: "es como me gusta verlos, con ese respeto de ser un individuo, no como un miembro de un grupo o parte de una estadística".

Durante su trayectoria académica, el doctor Eugenio ha publicado 23 artículos en revistas indexadas de circulación internacional, 18 en arbitradas y de divulgación, tres capítulos en libros de circulación internacional. ${ }^{4} \mathrm{Ha}$ impartido diez conferencias por invitación en eventos científicos, presentado sistemas de clonación que permitan multiplicar masivamente especies en peligro de extinción, de zonas áridas. Además, compartió que ha trabajado en: "el desarrollo de procesos biotecnológicos que nos permitan producir los compuestos químicos que se producen en cactáceas o plantas medicinales de forma natural en sistemas biotecnológicos [...] para evitar el daño a las poblaciones naturales que hay en el medio ambiente".

El doctor Eugenio compartió también cómo llegó a ser profesor: "mi gusto e intención al formarme, al estudiar, fue ser investigador, el ser profesor es parte inherente a la investigación científica, no se puede entender una sin la otra, y es algo que hago con agrado".

Asimismo, lo que más le gusta de la labor docente es: "el proceso de formación de alumnos dentro de la investigación. [...] Desde mi punto de vista, es una formación mucho más atractiva [...] que trasciende más que una clase solamente".
72 trabajos en congresos nacionales y 31 en internacionales. Como asesor de tesis ha dirigido 33 tesis y tesinas en el nivel licenciatura, 31 en maestría y seis en doctorado.
4 Pérez-Molphe-Balch, E. (2003). Genetic Transformation and Regeneration of Citrus Species. In. Pawan K. Jaiwal \& Rana P. Singh (Eds.). Plant Genetic Engineering, Vol. 6. Improvement of Fruit Crops. Sci-Tech Publishing LLC. Houston, uSA, 1-21; Nuñez-Palenius, H.G., Cantliffe, D.J., Klee, H.H., Ochoa-Alejo, N., Ramírez Malagón, R., PérezMolphe-Balch, E. (2006). Methods in Plant Tissue Culture. In. N.S. Pandian (Ed). Food Biotechnology, 2nd Edition. CRC Taylor \& Francis, New York, 553-601; Santos-Díaz, M. S., Pérez-Molphe, E., Ramírez-Malagón, R., Núñez-Palenius, H. G., Ochoa-Alejo, N. 2010. Mexican Threatened Cacti: Current Status and Strategies for their Conservation. In: Tepper, G.H. (Ed.). Species Diversity and Extinction. Nova Science Publishers, Inc. 1-60. 


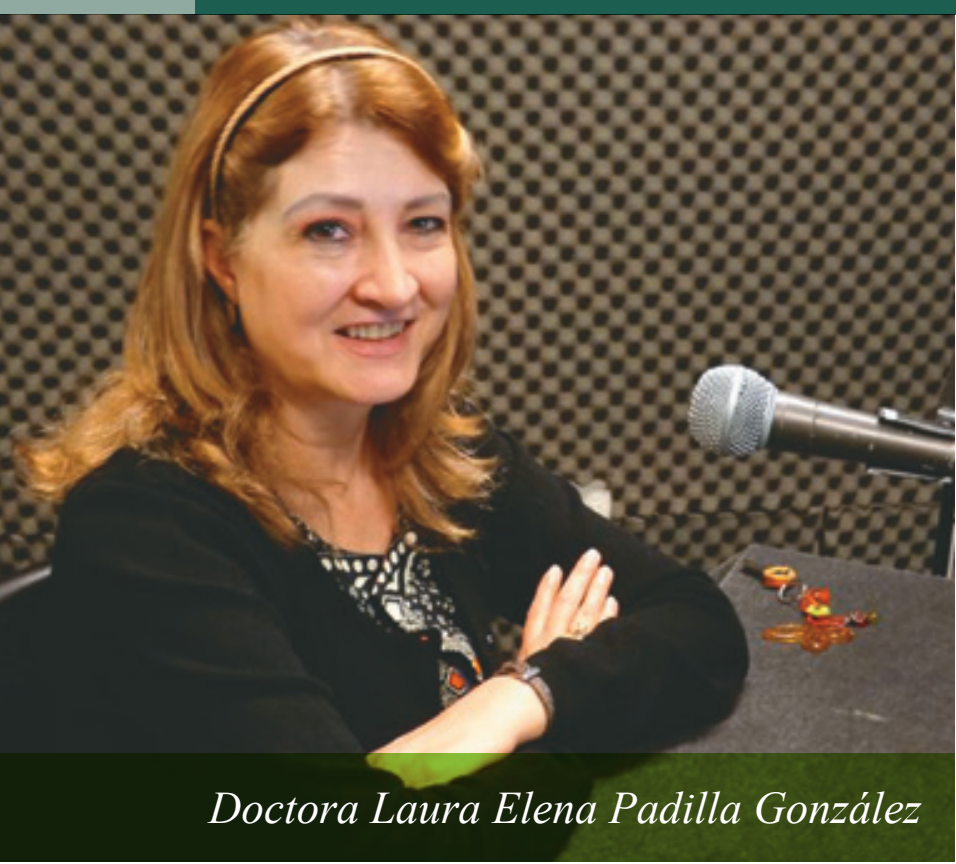

\section{Doctora Laura Elena Padilla González}

$\mathrm{P}$ rofesora investigadora de tiempo completo, adscrita al Departamento de Educación del Centro de Ciencias Sociales y Humanidades. Durante su trayectoria de 33 años en la institución, fue Directora General de Asuntos Académicos, que posteriormente se transformó en la Dirección General de Docencia de Pregrado; entre otros cargos $\mathrm{y}$ distinciones, pertenece al SNI.

En Aguascalientes, su ciudad natal, creció y realizó sus estudios de Licenciatura en Ciencias de la Educación, y posteriormente, la Maestría en Ciencias Sociales en la Universidad Autónoma de Aguascalientes. Con la inquietud de ampliar sus horizontes, se trasladó en 1999 a la ciudad de Claremont, en Los Ángeles, California, para realizar sus estudios de doctorado, obteniendo el título de Doctor of Philosophy in Education.

Su incursión en la docencia se dio en la época de 1980, en la que hubo un crecimiento de la educación superior en el país, generando una gran demanda de profesores para el nivel superior, por lo cual le tocó dar clase en el nivel técnico antes de graduarse de la licenciatura; e inmediatamente al egresar, incorporarse a la Dirección General de Asuntos Académicos (que años después le correspondería dirigir). En esta área se inició como asistente y luego Jefa del Departamento de Apoyo a la Investigación, en donde laboró bajo la dirección del Doctor Luis Manuel Macías López. Simultáneamente, colaboró con la formación docente, dado que un reto importante para la institución era ofrecer cursos a profesores; siendo esto fundamental en su inicio a la actividad académica en la institución.

Considerando la complejidad de la labor docente, la cual no implica únicamente la actividad frente a grupo comentó: "como académicos un reto importante es combinar las diversas funciones que uno tiene asignadas, ya que, se espera que además de la docencia, uno realice investigación y difusión del conocimiento, [...] además, del reto de seguirse formando para dar una atención integral a estas actividades".

Por otra parte, considera que:

[...] la responsabilidad de estar frente a un grupo en tu calidad de profesor siempre te plantea un reto, en cualquier etapa de tu vida [...]. [Sin embargo], en cualquier ámbito especialmente en la docencia y en la investigación, uno debe sentir el reto, el desafío [...] es la motivación para hacer mejor las cosas y para seguirse preparando [...]; entonces, que lo que uno haga se evidencie en ese aprendizaje de los estudiantes.

En cuanto a la incorporación de las tecnologías de la información y la comunicación, a los procesos de enseñanza y aprendizaje opina que: "un nuevo reto que los docentes tenemos es el incorporar las tecnologías, con base en las tendencias en las poblaciones estudiantiles [...]; sin perder de vista que el objetivo central es que los estudiantes aprendan a aprender, que adquieran el bagaje básico a partir del cual ellos puedan seguir aprendiendo de manera autónoma".

$\mathrm{Su}$ interés por la investigación comenzó desde sus estudios de licenciatura, siendo ésta una de las áreas de salida de la carrera por las cuales optar. Su incursión en la investigación se dio formalmente con sus estudios de maestría. Con un especial interés en el tema del desarrollo del personal académico en las instituciones de educación superior, por haber sido su ámbito inicial de trabajo, desarrolló su tesis sobre el Efec- $\ll$ Un nuevo reto que los docentes tenemos es el incorporar las tecnologías, con base en las tendencias

en las poblaciones estudiantiles; sin perder de vista que el objetivo central

es que los estudiantes aprendan a aprender, que adquieran el bagaje básico a partir del cual ellos puedan seguir aprendiendo de manera autónoma. $\gg$ 
to de los cursos de formación pedagógica en la práctica docente de los profesores universitarios, en colaboración con su colega y amiga la maestra Ma. de los Dolores Ramírez Gordillo.

A partir de su labor como investigadora, ha tenido la oportunidad de trabajar en redes académicas con otras universidades del país, lo que ha enriquecido su labor cotidiana en el aula, y su interacción académica con los estudiantes.

Entre sus publicaciones se encuentran dos libros como coautora, editados por la ANUIEs: Políticas públicas y cambios en la profesión académica en México en la última década, ${ }^{5}$ y Socialización de la nueva generación de investigadores en México ${ }^{6}$ este último fue fruto del trabajo de la red académica establecida entre la Universidad Autónoma Metropolitana, Universidad de Sonora y la UAA.

Además de otros artículos, tanto en el idioma inglés como en español, difundidos en revistas arbitradas e indexadas, algunos de ellos enfocados en el análisis del desarrollo de la mujer en la profesión académica; un ejemplo es el artículo Las mujeres en la profesión académica y el techo de cristal. Una perspectiva comparada en Norteamérica (México, Estados Unidos y Canadá), ${ }^{7}$ realizado a partir de su estancia académica en la University of British Columbia de Vancouver, Canadá, en colaboración con la profesora Amy Scott Metcalfe.

Lo que más disfruta de su trabajo es: "la emoción de lo nuevo, ésta es una carrera en la que siempre está uno aprendiendo, la investigación está generando nuevos conocimientos. [Por otra parte,] cada grupo es distinto, con sus propias inquietudes y situación, es algo que no te deja quedarte quieto, que te obliga a estar en constante cambio, que por eso mismo a veces es pesado, pero a la vez muy recompensante".

Finalmente, invita a los docentes a procurar su bienestar personal: "sepamos combinar nuestras

5 Grediaga, R. (Coord.). Rodríguez, J. y Padilla, L. (2004). Políticas públicas y cambios en la profesión académica en México en la última década. México: ANuiEs. Disponible en Google Books: http://bit.ly/1IN4tbV.

6 Grediaga, R. (Coord.). Hamui, M. y Padilla, L. (2012) Socialización de la nueva generación de investigadores en México. México: ANuies.

7 Padilla, L. y Metcalfe, A. (2012). Las mujeres en la profesión académica y el techo de cristal. Una perspectiva comparada en Norteamérica (México, Estados Unidos y Canadá). Caleidoscopio. Año 15, Núm. 27. México: UAA. actividades académicas con nuestra vida cotidiana, y con un espíritu positivo".

\section{Conclusiones}

La breve semblanza presentada en estas líneas, sobre la trayectoria de los tres docentes entrevistados, nos permitió un acercamiento al origen y desarrollo de su vida académica; a conocer un poco sobre las principales situaciones y decisiones que los llevaron a profesar en sus áreas de conocimiento como catedráticos de esta institución.

Además de conocer algunos de sus productos publicados, mismos que fueron desarrollados en el trabajo individual y colectivo, para irse consolidando como investigadores, y ser ahora promotores de la cultura y la ciencia en el ámbito local, nacional e internacional.

Finalmente, nos permitieron apreciar su pasión por la docencia y la investigación como actividades integradas a favor de la generación y difusión del conocimiento, y por supuesto, en beneficio de la formación integral de sus estudiantes, trabajo desarrollado por medio de esta máxima casa de estudios, la UAA.

\section{Fuentes de consulta}

Mateos, A. (2005). Compendio de etimologías grecolatinas del español. México: Esfinge.

Real Academia Española (2015). Diccionario. Recuperado el 1 de septiembre de 2015, en: http://bit.ly/1Jw26f8.

"Trayectorias docentes: doctora Consuelo Meza Márquez". "El Gis". UAA. Aguascalientes, Ags., 6 de marzo de 2014. Radio.

"Trayectorias docentes: doctor Eugenio Pérez Molphe Balch". "El Gis". UAA. Aguascalientes, Ags., 3 de abril de 2014. Radio.

"Trayectorias docentes: doctora Laura Elena Padilla González". "El Gis". UAA. Aguascalientes, Ags., 1 de mayo de 2014. Radio.

Meza, C. (1998). La radio como medio de sensibilización y difusión de la contracultura femenina. Recuperado el 3 de septiembre de 2015, disponible en: http://bit.ly/1Odx6E7.

UAA (2015). Proyecto radiofónico "El Gis". México: UAA (documento mecanoescrito). 